\title{
Organoorganoxysilane mit ringförmiger Struktur
}

\author{
Von Roshdy M. Ismail * \\ Aus dem Forschungslaboratorium der Firma Hans J. Zimmer, Frankfurt/Main
}

(Z. Naturforschg. 19 b, 873-874 [1964] ; eingegangen am 23. Januar 1964)

\begin{abstract}
Siebengliedrige Organoorganoxysilane zeichnen sich durch eine Reihe wertvoller Eigenschaften aus. Die Verbindungen werden durch Ringschluß substituierter difunktioneller Silane mit $2.2^{\prime}$-Diphenol erhalten.
\end{abstract}

Siebengliedrige siliciumorganische Verbindungen der allgemeinen Formel

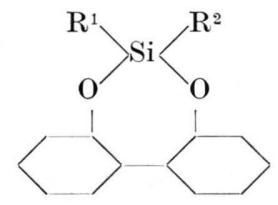

konnten in sehr guten Ausbeuten hergestellt werden. Die hochsiedenden, viskosen Verbindungen sind bei hohen Temperaturen gut destillierbar. Sie zeichnen sich durch thermische Stabilität, relativ gute Hydrolysebeständigkeit und einen tiefen Stockpunkt aus. Der Ringschluß mit 2.2'-Diphenol bewirkt infolge sterischer Hinderung ${ }^{1}$ gute Stabilität. Durch Variation der Organo- und Organoxygruppen R 1 und R 2 lassen sich die Eigenschaften beliebig modifizieren. Es ist bekannt, daß Tetraorganosilane außerordentlich stabil sind ${ }^{2}$. Sie übertreffen Tetraorganoxysilane in ihrer thermischen Beständigkeit ${ }^{3}$ und hydrolytischer Resistenz. In Organoorganoxysilanen verhalten sich niedrige Alkoxygruppen siliciumfunktionell. Sie ähneln in ihrem chemischen Verhalten Halogenresten und können wie diese Substitutionsreaktionen eingehen. Sie sind demzufolge sehr hydrolyseempfindlich. Mit zunehmender Größe des Organo- oder Organoxyrestes erhöht sich jedoch die Hydrolyseresistenz und die thermische Beständigkeit.

Als Ausgangsstoffe zur Herstellung beständiger Organorganoxysilane dienen 2.2'-Diphenol, aliphatische oder aromatische Hydroxyverbindungen und di- oder trifunktionelle Organosilane. Die Umsetzung kann durch Umesterung oder Kondensation erfolgen. Geringe Mengen der Alkalisalze der Hydroxyver-

* unter Mitarbeit von P. Olschewsin und B. Gellenbeck.

1 H. S. Nametrin, A. V. Topschisew u. F. F. Matschus, Ber. Akad. Wiss. UdSSR 83, 705 [1952].

2 W. L. Ipatiev u. B. Dolgov, Ber. dtsch. chem. Ges. 62, 1220 [1929].

3 G. Fritz u. B. RaABe, Z. anorg. allg. Chem. 286, 149[1956]. bindungen beschleunigen die Umesterungsreaktion katalytisch ${ }^{4}$. Alle Verbindungen lassen sich bei höheren Temperaturen unzersetzt destillieren. Sind hingegen im Molekül ausschließlich Si-O-C-Bindungen enthalten, so kann im Verlaufe der Destillation Umesterung erfolgen ${ }^{5}$. Bei der Destillation von Diorganoxydiphendioxysilanen scheidet sich der Kieselsäureester des 2.2'-Diphenols als hochschmelzende (Schmp. $322{ }^{\circ} \mathrm{C}$ ) weiße Verbindung ab:

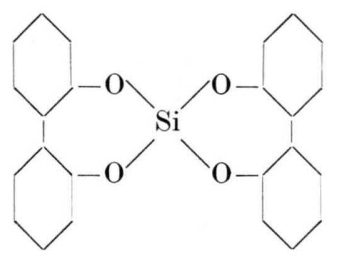

Analyse: Gef. C $73,1 \quad$ H 4,3 Si 7,2 ,

Ber. C 72,7 H 4,1 Si 7,1 .

Die hergestellten Verbindungen eignen sich u. a. als Wärmeübertragungsmittel ${ }^{6,7}$ und hydraulische Flüssigkeiten ${ }^{8}$.

Es wurden folgende Verbindungen dargestellt: Diphenyl-diphendioxy-silan (I), Dimethyl-diphendioxy-silan (II), Phenyl-octoxy-diphendioxy-silan (III), Phenyl-decoxy-diphendioxy-silan (IV), Phenyl2-methyl-4-tert.-hexylphenoxy-diphendioxy-silan (V), Phenyl - phenoxy - diphendioxy - silan(VI), Phenylcyclododecoxy-diphendioxy-silan (VII), Phenyl-1methyl - 4 - äthylhexoxy - diphendioxy - silan (VIII), Phenyl-1.4-diäthylhexoxy-diphendioxy-silan (IX), Phenyl-1-methyl-4-äthyloctoxy-diphendioxy-silan(X), Phenyl-1.4-diäthyloctoxy-diphendioxy-silan (XI) .

Die Analysen und Eigenschaften der Verbindungen ergeben sich aus Tab. 1 .

4 R. M. Ismail, Z. Naturforschg. 18 b, 582 [1963].

5 R. M. Ismail, Z. Naturforschg. 18 b, 1122 [1963].

6 U.S.-Pat. 2.674.579.

7 Brit.-Pat. 696.237.

8 U.S.Pat. 2.701.803. 


\begin{tabular}{|c|c|c|c|c|c|c|c|c|c|c|c|}
\hline \multirow{3}{*}{$\begin{array}{c}\text { Ver- } \\
\text { bindung }\end{array}$} & \multicolumn{6}{|c|}{ Analysen } & \multicolumn{4}{|c|}{ Physikalische Daten } & \multirow{3}{*}{$\begin{array}{c}\text { Ausbeute } \\
{[\%]}\end{array}$} \\
\hline & \multicolumn{2}{|c|}{$\mathrm{C}$} & \multicolumn{2}{|c|}{$\mathrm{H}$} & \multicolumn{2}{|c|}{$\mathrm{Si}$} & \multirow[t]{2}{*}{$\begin{array}{l}\text { Sdp. } \\
{\left[{ }^{\circ} \mathrm{C}\right]}\end{array}$} & \multirow[t]{2}{*}{$\begin{array}{c}\text { Druck } \\
{[\mathrm{mm}]}\end{array}$} & $n_{20}^{\mathrm{D}}$ & \multirow[t]{2}{*}{$n_{70}^{\mathrm{D}}$} & \\
\hline & Gef. & Ber. & Gef. & Ber. & Gef. & Ber. & & & & & \\
\hline I & 78,2 & 78,7 & 4,7 & 5,0 & 7,9 & 7,7 & $235-236$ & 0,5 & (fest: $\mathrm{F}$ & $\left.40^{\circ} \mathrm{C}\right)$ & 83 \\
\hline II & 69,3 & 69,4 & 5,7 & 5,8 & 11,6 & 11,6 & 136 & 2,5 & (fest: F & $\left.124^{\circ} \mathrm{C}\right)$ & 91 \\
\hline III & 74,1 & 74,6 & 6,9 & 7,2 & 6,8 & 6,7 & $230-235$ & 0,8 & 1,5662 & - & 85 \\
\hline IV & 75,9 & 75,3 & 7,2 & 7,7 & 6,5 & 6,3 & $241-245$ & 0,8 & 1,5556 & - & 80 \\
\hline V & 77,0 & 77,5 & 6,5 & 6,7 & 6,0 & 5,8 & $275-280$ & 1,5 & - & 1,5728 & 82 \\
\hline VI & 75,3 & 75,4 & 4,8 & 4,7 & 7,4 & 7,3 & $228-230$ & 1,5 & 1,5451 & - & 80 \\
\hline VII & 76,0 & 76,2 & 7,6 & 7,7 & 5,9 & 5,9 & $297-302$ & 2,5 & - & 1,5615 & 91 \\
\hline VIII & 74,5 & 75,0 & 7,3 & 7,5 & 6,6 & 6,5 & $210-216$ & 0,2 & 1,5488 & - & 84 \\
\hline IX & 74,9 & 75,3 & 7,5 & 7,7 & 6,4 & 6,3 & $220-224$ & 0,3 & 1,5484 & - & $\begin{array}{l}89 \\
89\end{array}$ \\
\hline $\mathrm{X}$ & 75,2 & 75,6 & 8,1 & 7,9 & 6,3 & 6,1 & $226-228$ & 0,3 & 1,5418 & - & 83 \\
\hline XI & 75,4 & 75,9 & 8,3 & 8,1 & 6,1 & 5,9 & $235-236$ & 0,6 & 1,5400 & - & 87 \\
\hline
\end{tabular}

Tab. 1. Analysen und Eigenschaften der Verbindungen.

\section{Praktischer Teil}

\section{Diphenyl-diphendioxy-silan}

Zu 50,6 g (0,20 Mol) Diphenyldichlorsilan werden unter stürmischem Rühren rasch $16,0 \mathrm{~g}(0,50 \mathrm{Mol})$ abs. Methanol getropft. Durch zweistündiges Erhitzen am Rückfluß unter Durchleiten von trockenem Stickstoff wird die Reaktion vervollständigt. Nach Zugabe eines innigen Gemisches aus 37,05 g (0,199 Mol) 2.2'-Diphenol und $0,23 \mathrm{~g}(0,001 \mathrm{Mol})$ des entsprechenden Dinatriumsalzes wird für eine weitere Stde. unter Rühren erhitzt. Das entstehende Methanol wird abgezogen und der Rückstand unter Vakuum destilliert. Diphenyldiphendioxy-silan geht bei $235-236{ }^{\circ} \mathrm{C} \quad(0,5 \mathrm{~mm})$ über. Ausbeute: $61 \mathrm{~g}(83 \%)$.

\section{Phenyl-cyclododecoxy-diphendioxy-silan}

$42,3 \mathrm{~g} \quad(0,20 \mathrm{Mol})$ Phenyltrichlorsilan und 52,2 $\mathrm{g}$ (0,66 Mol) Pyridin werden in $250 \mathrm{ml}$ abs. Benzol gelöst und unter Rühren tropfenweise mit einer konzentrierten benzolischen Lösung von $36,9 \mathrm{~g}$ (0,20 Mol)
Cyclododecanol versetzt. Durch einstündiges Kochen am Rückfluß wird die Reaktion beendet. Nach tropfenweiser Zugabe einer konzentrierten benzolischen Lösung von $37,2 \mathrm{~g} \quad(0,20 \mathrm{Mol}) 2.2^{\prime}$-Diphenol wird eine weitere Stde. am Rückfluß erhitzt. Das erkaltete Reaktionsgemisch wird filtriert, anschließend zentrifugiert und nach Abziehen des Lösungsmittels unter Vakuum destilliert. Phenyl-cyclododecoxy-diphendioxy-silan geht bei $297-302{ }^{\circ} \mathrm{C} \quad(2,5 \mathrm{~mm})$ über. Ausbeute: $86 \mathrm{~g}$ (91\%).

\section{Phenyl-1-methyl-4-äthylhexoxy-diphendioxy-silan}

$16,6 \mathrm{~g}(0,10 \mathrm{Mol})$ des Natriumsalzes von 5-Äthylheptanol-2 werden in $100 \mathrm{ml}$ abs. Toluol gegeben und beim Siedepunkt des Lösungsmittels unter Rühren tropfenweise mit 21,2 g (0,10 Mol) Phenyltrichlorsilan versetzt. Nach $2-4 \mathrm{Stdn}$. werden $23,0 \mathrm{~g}(0,10 \mathrm{Mol})$ des Dinatriumsalzes von 2.2'-Diphenol zugegeben. Es wird 2-4 Stdn. am Rückfluß gekocht. Toluol wird abgezogen und der Rückstand unter Vakuum destilliert. Phenyl-1-methyl-4-äthyl-hexoxy-diphendioxy-silan geht bei $210-216^{\circ} \mathrm{C}(0,2 \mathrm{~mm})$ über. Ausbeute: $36,5 \mathrm{~g}$ $(84 \%)$. 